\title{
Observatorio
}

\section{Cooperación bibliotecaria para un acceso global al conocimiento}

\author{
Por María-Antonia Carrato-Mena
}

Resumen: El acceso global al conocimiento es uno de los retos más interesantes para los bibliotecarios en el momento actual. La cooperación en red en el ámbito local, regional, nacional e internacional es imprescindible para la consecución de este logro. La política bibliotecaria del Ministerio de Cultura en estos últimos años ha permitido crear las estructuras necesarias de cooperación para que todas las administraciones y todo tipo de bibliotecas puedan colaborar entre sí con proyectos e iniciativas encaminadas a un desarrollo conjunto de sus redes y sistemas, que permita el reconocimiento de las bibliotecas como instituciones democráticas clave en las políticas educativas, culturales, sociales, de investigación y desarrollo.

Palabras clave: Cooperación bibliotecaria, Ministerio de Cultura, España, Proyectos de cooperación, Políticas, Cooperación internacional.

\section{Title: Library cooperation for global access to knowledge}

Abstract: Global access to knowledge is one of the most interesting challenges for librarians at the moment. Network cooperation at the local, regional, national and international level is essential to achieve this goal. The Ministry of Culture's library policy in recent years has allowed for the creation of the needed cooperation structures so that all administrations and all types of libraries can collaborate together in projects and initiatives targeted at the joint development of their networks and systems, which provides for the acknowledgement of libraries as key democratic institutions in education, cultural, social and research and development policies.

Keywords: Library cooperation, Ministry of Culture, Spain, Cooperation projects, Policies, International cooperation.

Carrato-Mena, María-Antonia. "Cooperación bibliotecaria para un acceso global al conocimiento". El profesional de la información, 2010, septiembre-octubre, v. 19, n. 5, pp. 449-454.

DOI: $10.3145 /$ epi.2010.sep.01

EN UN MUNDO GLOBALIZADO Y EN CONSTANTE CAMBIO, necesitado de creatividad e iniciativas innovadoras para superar el momento crítico que atraviesa, las bibliotecas adquieren el interesante reto de garantizar el acceso global al conocimiento.

Organismos internacionales como IFLA, sensibles a la necesidad de articular las acciones que conduzcan al logro de este objetivo, organizan debates y encuentros en torno a este tema sobre la premisa indispensable de la cooperación. Se subraya la importancia de construir redes, alianzas, infraestructuras técnicas, sociales, políticas y educacionales para lograr una sociedad inclusiva con acceso global al conocimiento en todos los niveles: local, regional, nacional e internacional.

La acción de cooperar, voluntaria por definición, lleva siempre implícito un acto de comunicación, mediante relaciones horizontales entre las personas e instituciones que deciden colaborar entre sí, a través de canales que se han ido multiplicando de forma exponencial gracias a las TIC. La cooperación en red surge por múltiples razones y en muy diferentes entornos pero habitualmente se produce con el fin de compartir, rentabilizar recursos y conseguir un mayor nivel de servicios (en cantidad y calidad) y una mayor visibilidad.

El incremento excepcional del número de publicaciones y contenidos en la Red junto al cambio 
tecnológico que se ha producido en las últimas décadas, utilizando internet como recurso y medio en los procesos de información y comunicación, han supuesto una transformación total en la forma en que los bibliotecarios organizan, gestionan y facilitan el acceso a la información y, consecuentemente, en la posibilidad de generar nuevo conocimiento.

La cooperación bibliotecaria en red es esencial si se pretende la ampliación, la mejora, la innovación y una mayor competitividad en la

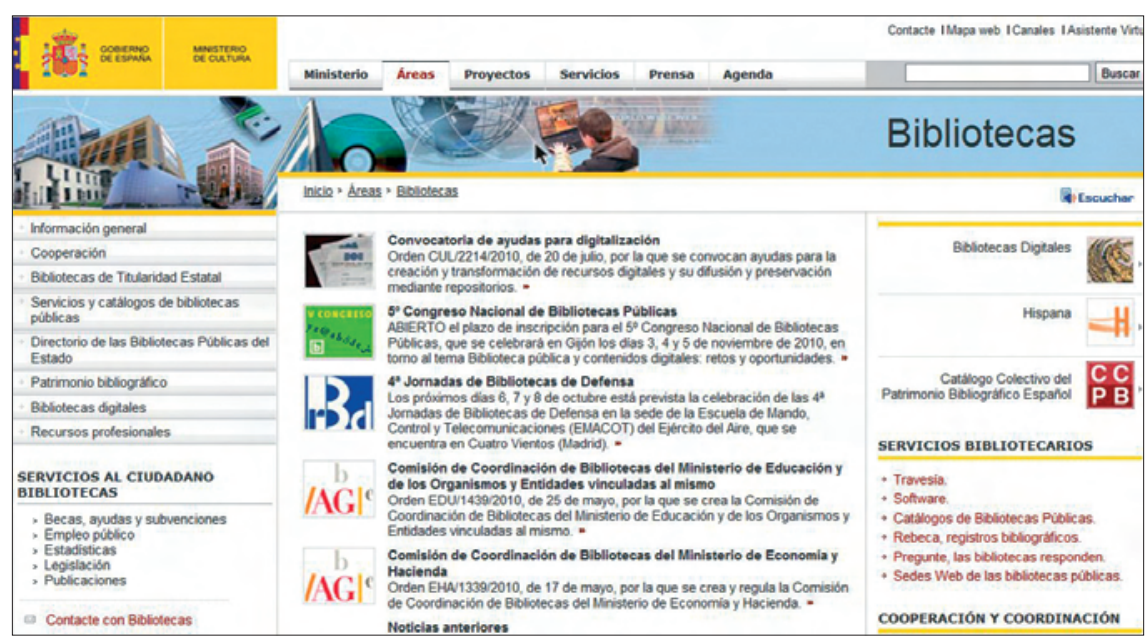

SG de Coordinación Bibliotecaria, Ministerio de Cultura http://www.mcu.es/bibliotecas/ todo tipo se integran en redes, sistemas o consorcios con el fin de alcanzar un grado de eficiencia superior al que lograrían trabajando aisladamente. Desde la creación de redes bibliotecarias para la localización de contenidos y facilitar el préstamo bibliotecario a partir de catálogos colectivos, al establecimiento de redes cooperativas para la creación de bibliotecas digitales, plataformas educativas o proyectos con fines de preservación, se ha recorrido un largo camino, en el que también se han entrecruzado las redes sociales, favoreciendo el desarrollo de las relaciones personales y comunidades de interés y proporcionando una mayor visibilidad a las bibliotecas en los nuevos espacios de comunicación.

\section{"La cooperación tiene aún más sentido en España, con una estructura político- administrativa descentralizada y un reparto competencial distribuido entre las diferentes administraciones"}

En los diferentes modelos de redes según su estructura, ámbito administrativo o geográfico, materia o fines objeto de la cooperación, se pueden generar sinergias que siempre tienen efectos positivos en el resultado final. La posibilidad de colaborar con otros actores de sectores no bibliotecarios, públicos y privados, es una oportunidad que en ocasiones puede ser decisiva para poner en marcha redes cooperativas.

Si la cooperación es siempre necesaria, aún tiene más sentido en un país como España, con una estructura político-administrativa descentralizada y un reparto competencial en materia bibliotecaria distribuido entre las diferentes administraciones que lo integran. Esta estructura no supone en principio una desventaja si se planifica la colaboración de forma integrada y se establecen los mecanismos que garanticen la cooperación entre todas las administraciones y sectores implicados.

\section{Papel de la SGCB}

En los últimos años, desde la Subdirección General de Coordinación Bibliotecaria (SGCB) se ha llevado a cabo una estrategia con acciones que, de forma progresiva, han permitido la creación de estructuras de cooperación y promovido la colaboración entre las bibliotecas: nacionales, universitarias, escolares, públicas y especializadas.

En el ámbito bibliotecario se había producido siempre la colaboración profesional, pero hasta la aprobación de la Ley de la lectura, del libro y de las bibliotecas en el año 2007 no se contemplaba un marco global que estructurara esa cooperación y aunara a bibliotecarios y políticos en un mismo órgano con representación de todas las administraciones y de todo tipo de bibliotecas.

Los desarrollos reglamentarios de esta Ley, que se elaboraron de forma consensuada con todos los actores implicados, han permitido la puesta en funcionamiento no sólo del Consejo de Cooperación Bibliotecaria, órgano que canaliza la cooperación interadministrativa, sino también de la Comisión General de Coordinación de las bibliotecas de la Administración General del Estado y de sus organismos públicos, nada menos que 879 bibliotecas, que a su vez son coordinadas por sus respectivas comisiones ministeriales.

En el proceso de constitución de esas estructuras administrativas se han incorporado redes y proyectos anteriores a la citada Ley, al tiempo que se ha creado el marco necesario para nuevas propuestas y acciones de colaboración. En este espacio se producen debates y consensos, intercambio de conocimiento, toma de decisiones, propuesta de normativa, de documentos de uti- 


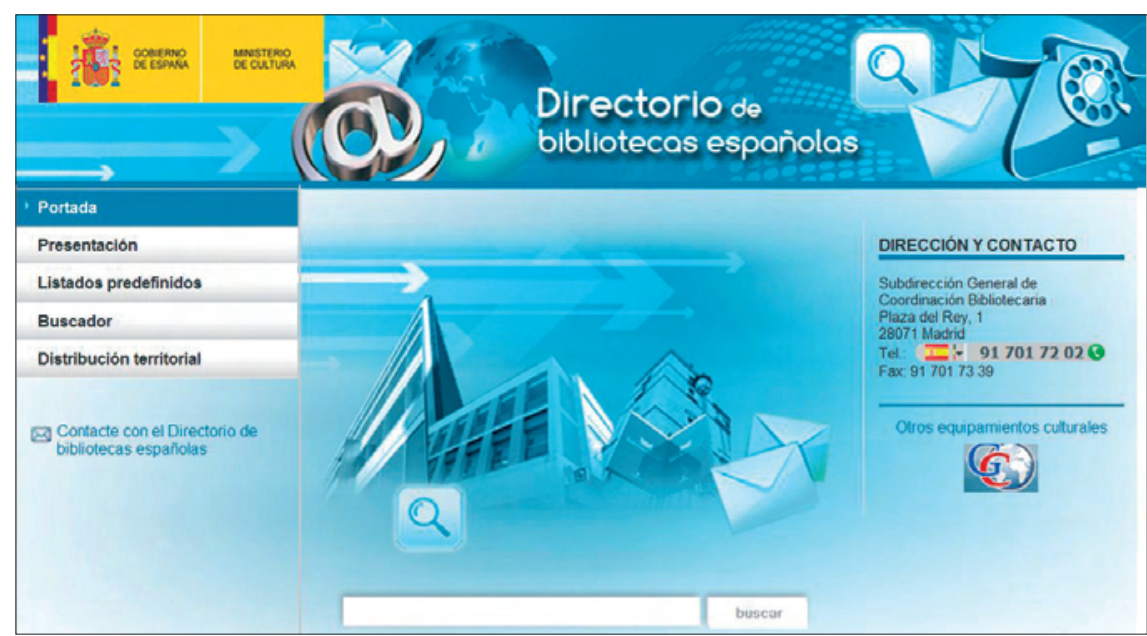

Directorio de bibliotecas españolas http://directoriobibliotecas.mcu.es/

lidad para las bibliotecas, inicio de nuevos proyectos, etc. Como no podía ser de otra forma, una parte fundamental de esta actividad se realiza mediante la comunicación sobre diferentes aplicaciones que facilitan la cooperación en red entre profesionales y la interconexión entre personas con estrategias e intereses comunes.

La actividad del Consejo dispone de una sede colaborativa de trabajo sobre una aplicación de código abierto desarrollada por la Subdirección General de Tecnologías y Sistemas de la Información del Ministerio de Cultura $(M C U)$. Son en este momento 500 los usuarios, que pertenecen a 15 grupos de trabajo, a las cinco comisiones técnicas de cooperación, a la Comisión Permanente, a la Secretaría Permanente y al Pleno, los que interactúan en esta sede web a través de foros de comunicación interna para conocer las últimas novedades; participar en las líneas de debate abiertas; compartir documentos de trabajo; recibir noticias actualizadas por RSS; realizar reuniones virtuales mediante chat en tiempo real y emitir su voto, entre otras opciones.

"La colaboración con sectores no bibliotecarios, públicos y privados, es una oportunidad que en ocasiones puede ser decisiva"

Toda esta información y comunicación interna entre los distintos órganos tiene una parte visible para el resto de profesionales interesados y para el ciudadano en la web del Consejo de Cooperación Bibliotecaria, donde se informa sobre datos relativos a los miembros que forman parte de los distintos órganos, se publican los documentos aprobados por el Consejo, los acuerdos alcanzados en las reuniones, los objetivos y líneas de trabajo, etc., contribuyendo de esta manera a la transparencia en la actuación de los órganos de la Administración.

En lo que respecta a la coordinación de las bibliotecas de la Administración General del Estado, el Real Decreto 1572/2007 regula la composición de los órganos que se crean con el fin de garantizar la idoneidad de los procesos desarrollados y los servicios prestados, así como establecer un punto de consulta único de todas sus colecciones, de enorme riqueza (artículo 1.2 , letras a) y b)).

\section{Comisiones ministeriales}

La constitución de las comisiones ministeriales se ha ido produciendo de manera progresiva mediante la publicación en el $B O E$ de las correspondientes órdenes. Por su parte, la Secretaría Permanente de la Comisión General de Coordinación consideró conveniente realizar un estudio previo, mediante una encuesta, que permitiese conocer la situación real de las bibliotecas. A los efectos de los objetivos establecidos en el Real Decreto 1572/2007, los datos más relevantes obtenidos de este estudio fueron que cerca del $40 \%$ de las bibliotecas de la Administración General del Estado y de sus organismos públicos no estaban automatizadas, lo cual suponía que el $25 \%$ de sus colecciones no eran accesibles a través de catálogos automatizados.

En la primera reunión de la Comisión General de Coordinación, el 22 de septiembre de 2009, se acordó la creación de un Grupo de Trabajo, con representación de todos los ministerios, con el objetivo de poner en marcha el punto de consulta único de las colecciones de las bibliotecas de la Administración General de Estado (AGE) y de sus organismos públicos y de automatizar aquellas bibliotecas que aún no han abordado este proceso. Desde su creación, el Grupo trabaja en la evaluación y selección entre programas de código abierto. Esta decisión supone una apuesta por este tipo de programas para su implementación progresiva de forma conjunta, al tiempo que promueve la participación de una diversidad de empresas en el transcurso del proyecto.

\section{Cooperación}

Uno de los objetivos estratégicos de la Subdirección General de Coordinación Bibliotecaria (SGCB) es la política de cooperación en primer lugar con las comunidades autónomas, pero también con el resto de 


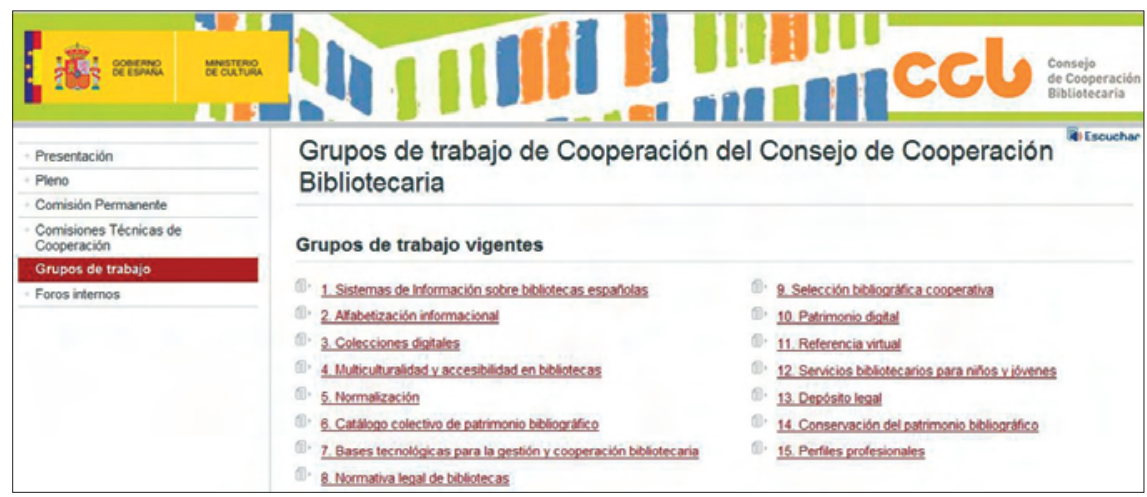

Consejo de Cooperación Bibliotecaria

http://www.mcu.es/bibliotecas//MC/ConsejoCB/Presentacion.html
- oferta de servicios de referencia en línea a los ciudadanos, como el proyecto cooperativo Pregunte: las bibliotecas responden;

- prestación de servicios que permitan a las bibliotecas hacer accesibles sus contenidos en internet, como el Generador de sedes web, o difundir el patrimonio arquitectónico de las bibliotecas, como el proyecto Arquitectura y bibliotecas; y

- establecimiento de foros de encuentro, difusión y promoción de las administraciones así como con las asociaciones y otros agentes del sector.

Esta cooperación, que va creciendo, está produciendo importantes resultados en muchas áreas y demuestra que el diálogo y la cooperación, siempre sujetos al entorno normativo europeo e internacional, son la mejor de las soluciones para el planteamiento de proyectos que a medio y largo plazo. Producen buenos resultados y repercuten muy favorablemente en el acceso de los ciudadanos a la educación, la cultura y la información; en la preservación y difusión del patrimonio bibliográfico español; y en el proceso de alfabetización digital y de desarrollo de competencias en el manejo de la información. De esta forma vamos reduciendo la brecha que nos separa de los países más avanzados en el uso de las tecnologías de la información y la comunicación, cumpliendo así el objetivo esencialmente democrático de las bibliotecas.

\section{Proyectos del Ministerio de Cultura}

El $M C U$ está haciendo un especial hincapié y esfuerzo, tanto técnico como presupuestario, para avanzar en distintos proyectos cooperativos en la Red, muchos de ellos resultado de la actividad cooperativa de los grupos de trabajo del Consejo, antes mencionados.

Estos proyectos incluyen, entre otros:

- catalogación cooperativa en red, como Rebeca;

- elaboración y mantenimiento cooperativos del Catálogo del Patrimonio Bibliográfico Español;

- digitalización de los materiales bibliográficos de titularidad pública y privada, para lo que ha puesto en producción la Biblioteca Virtual de Prensa Histórica y la Biblioteca Virtual del Patrimonio Bibliográfico;

- creación de recursos digitales que aseguren no sólo la preservación de los contenidos sino también su accesibilidad y visibilidad en internet, mediante las resoluciones por las que se convocan ayudas para proyectos de digitalización; actividades para mejorar competencias informacionales relacionadas con diferentes situaciones y entornos sociales como la plataforma Alfared.

La cooperación en red entre las instituciones de la memoria, archivos, bibliotecas y museos, responde a otra de las líneas de actuación cooperativa del $M C U$, con la finalidad de ofrecer de forma conjunta al ciudadano los contenidos culturales en internet.

\section{Estadísticas}

La recogida de datos cuantitativos de bibliotecas para realizar el análisis y la evaluación de la actividad bibliotecaria es fundamental. Por ello, y cumpliendo con la función que tiene asignada el Ministerio de Cultura según el Real Decreto 1132/2008, la SGCB, en el marco de cooperación del Grupo de trabajo del Sistema de Información sobre Bibliotecas Españolas del Consejo, colabora con todas las administraciones y entidades titulares o con competencias sobre bibliotecas. Ofrece una aplicación informática en la que se realiza la carga de datos en un cuestionario previamente consensuado mediante acceso directo de cada biblioteca, centralizado desde los servicios de bibliotecas autonómicos o unidades de coordinación de la AGE o bien realizando una carga masiva de datos en ficheros previamente parametrizados.

Está previsto instalar en breve un módulo de control y calidad de los datos y otro de explotación en línea, que permitirán evaluar los recursos, procesos y servicios de las bibliotecas de forma rápida y sencilla y ofrecerá la posibilidad de consultar los datos absolutos de una biblioteca y/o red de bibliotecas individualizada, de una ciudad, provincia o comunidad autónoma; análisis porcentuales; variaciones interanuales; análisis comparativos respecto a años anteriores o entre bibliotecas, poblaciones y regiones.

En la actualidad la Subdirección centra sus esfuerzos en acortar los plazos de recogida y publicación de los resultados así como en la adaptación del Directorio de bibliotecas, realizado en cooperación con las comu- 
nidades autónomas, a la norma ISO 2146. El directorio marco sirve tanto de base para la recogida de datos que lleva a cabo el Instituto Nacional de Estadística (INE) como para planificar toda la actividad que realiza la $S G C B$.

\section{Cooperación internacional}

Si nos trasladamos al plano de cooperación internacional, es evidente que en estos últimos años se observa una mayor presencia de profesionales españoles en los foros europeos e internacionales. Esto facilita los contactos personales con otros países que, en ocasiones, de-

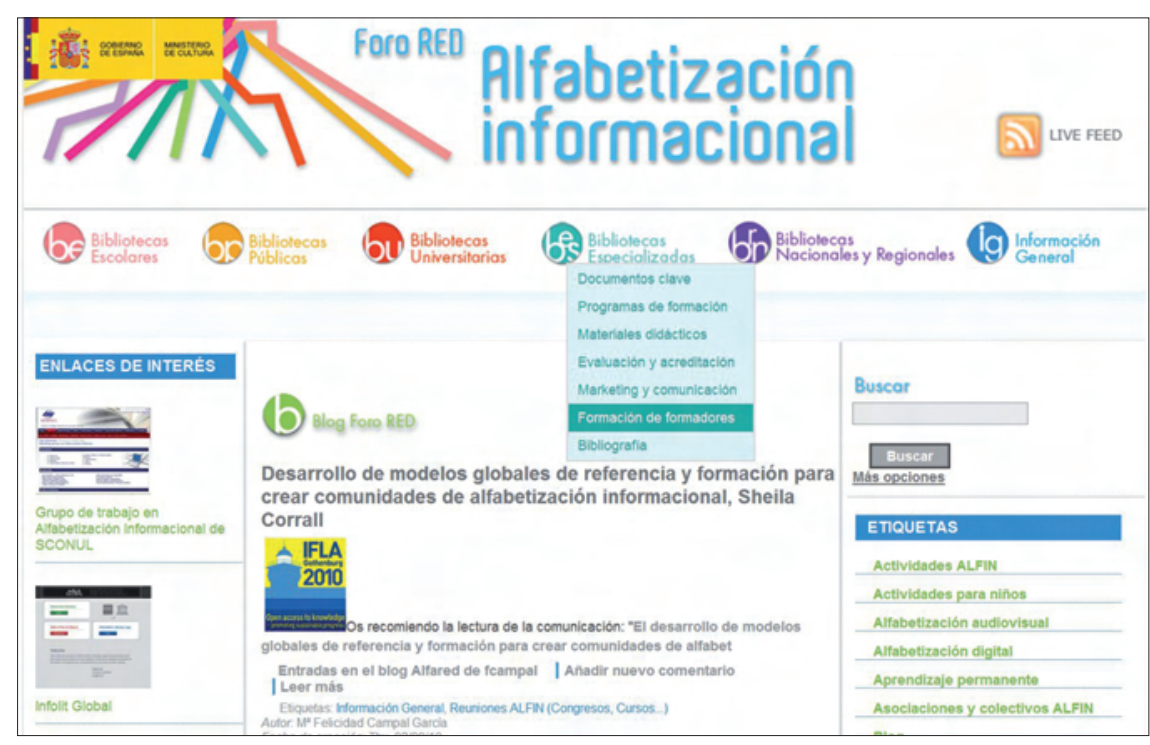

http://www.alfared.org/ rivan en la realización de proyectos conjuntos en red. Sería deseable una mayor implicación en este tipo de iniciativas que, si bien es cierto que requieren esfuerzo y tiempo, revierten de forma positiva en la extensión de la cooperación más allá de nuestro espacio habitual.

Desde los foros europeos e internacionales en los que participa, el $M C U$ ha promovido iniciativas de cooperación, proponiendo una reformulación del programa Picbip en el Firbip (Foro iberoamericano de responsables nacionales de bibliotecas públicas) o propiciando por primera vez una estrecha colaboración entre Naple (National authorities for public libraries in Europe), foro que preside desde 2007, y Eblida, en temas relacionados con la propiedad intelectual o en la redacción de un libro blanco para las bibliotecas públicas en el seno de la Comisión Europea.

Ha multiplicado también su actividad y participación en IFLA y en el proyecto Europeana, estableciendo de forma paralela relaciones de cooperación con países como Alemania o Finlandia. Estas acciones han permitido, entre otras cosas, dar a conocer el grado de evolución de las bibliotecas españolas y facilitado su incorporación a proyectos europeos con un alto nivel de participación, en especial en lo que a biblioteca digital se refiere.

En este último aspecto, y siempre en cooperación con las distintas administraciones e instituciones privadas, titulares de una gran parte del patrimonio bibliográfico español, se lleva a cabo un doble esfuerzo para la creación de recursos digitales consistentes en la digitalización de los materiales bibliográficos y en la asignación de metadatos que faciliten la recolección de la información en la Red, su máxima visibilidad en los buscadores más importantes e, incluso, dar los primeros pasos en una política de preservación digital.
La implementación de Hispana, directorio y recolector de colecciones y recursos digitales basado en el protocolo $O A I-M P H$, pretende asegurar en la medida de lo posible la máxima coordinación de los proyectos de digitalización en España y, simultáneamente, el acceso a casi 2.500.000 registros digitalizados (a fecha julio de 2010).

La normalización es imprescindible en cualquier proyecto de cooperación en red. La convocatoria anual de ayudas para la creación o transformación de recursos digitales, que el $M C U$ publica desde 2007, ha sido crucial en este caso para fomentar la creación de nuevas colecciones y recursos digitales en acceso abierto.

Las normativas y protocolos promovidos por la $S G C B$ se corresponden con las tendencias generales de la biblioteconomía internacional, y permiten la interoperabilidad y la cooperación con proyectos nacionales e internacionales. Son de obligado cumplimiento en el entorno de la iniciativa del proyecto Europeana al que Hispana ha aportado ya 1.250 .000 registros, situándose así España como uno de los primeros proveedores de contenidos. La aportación española a Europeana procede de bibliotecas digitales regionales y locales, de algunas instituciones privadas, de las citadas bibliotecas virtuales de la $S G C B$ y del proyecto Cer.es: colecciones en red (Red Digital de Colecciones de Museos de España).

\section{Conclusión}

El futuro nos aportará nuevas formas para crear contenidos de calidad y difundirlos en la Red de forma interactiva con los usuarios. Durante casi dos décadas el sector bibliotecario colaboró en la consecución del objetivo del acceso universal a las publicaciones como una de sus mejores aportaciones a la sociedad. Continuar profundizando en la idea de utilizar la cooperación 
bibliotecaria en red para ofrecer un acceso global al conocimiento sin duda es un reto que ayudará a eliminar barreras en el desarrollo equilibrado de esta sociedad.

Las posibilidades que ofrece una red bibliotecaria bien articulada son importantes, ya que pueden traducirse en oportunidades para la resolución de la brecha digital; la cultura; la educación; la investigación; la formación a lo largo de la vida; la creatividad; la innovación y la participación ciudadana. La formación permanente de los bibliotecarios y profesionales de la información, actores principales en este proceso, es condición indispensable para garantizar su liderazgo en la sociedad del conocimiento.

\section{Referencias}

Albsjer, Peter. "Interaction: anything goes 2.0". Scandinavian public libraries quaterly, 2008, v. 41, n. 2.

\section{Alfared}

http://www.alfared.org

Arquitectura y bibliotecas

http://www.mcu.es/arquitecturaybibliotecas

Bevan, Paul. "Share. Collaborate. Innovate. Building an organizacional approach to web 2.0". Ariadne, 2009, octubre, n. 61.

Bibliotecas de la Administración General del Estado y de sus organismos públicos

http://www.mcu.es/bibliotecas/MC/BAGE/index.html

Biblioteca virtual de prensa histórica

http://prensahistorica.mcu.es

Biblioteca virtual del Patrimonio Bibliográfico Español http://bvpb.mcu.es

Catálogo colectivo del patrimonio bibliográfico español http://www.mcu.es/bibliotecas/MC/CCPB

Cathro, Warwick. Collaboration experiences for digital collections: the Australian experience.

http://www.nla.gov.au/openpublish/index.php/nlasp/article/view/1433/1738

Cer.es, Red Digital de Colecciones de Museos de España

http://ceres.mcu.es

Consejo de Cooperación Bibliotecaria

http://www.mcu.es/bibliotecas//MC/ConsejoCB/Presentacion.html
Directorio de bibliotecas españolas http://directoriobibliotecas.mcu.es/

Directorio de bibliotecas de titularidad estatal. Madrid: Ministerio de Cultura, 2008.

http://www.mcu.es/bibliotecas/MC/DBTE/Presentacion.html

España. Ley 10/2007, de 22 de junio, de la lectura, del libro y de las bibliotecas. Boletín oficial del estado, 23 de junio de 2007, n. 150, pp. 27140-27150. http://www.boe.es/boe/dias/2007/06/23/pdfs/A27140-27150.pdf

España. Real Decreto 1573/2007, de 30 de noviembre, por el que se aprueba el Reglamento del Consejo de Cooperación Bibliotecaria. Boletín oficial del estado, 1 de enero de 2008, núm. 1, p. 7-12.

http://www.boe.es/boe/dias/2008/01/01/pdfs/A00007-00012.pdf

España. Real Decreto 1572/2007, de 30 de noviembre, por el que se regulan los órganos de coordinación de las bibliotecas de la Administración General del Estado y de sus organismos públicos. Boletín oficial del estado, 1 de enero de 2008, núm. 1, p. 4-7.

http://www.boe.es/boe/dias/2008/01/01/pdfs/A00004-00007.pdf

Firbip (Foro iberoamericano de responsables nacionales de bibliotecas públicas)

http://www.iberbibliotecas.org/

Generador de sedes web para bibliotecas públicas

http://www.bibliotecaspublicas.es

Hispana: directorio y recolector de recursos digitales http://roai.mcu.es

Naple (National authorities for public libraries in Europe) http://www.mcu.es/naple/index.html

Pregunte, las bibliotecas responden

http://www.pregunte.es

Rebeca

http://www.mcu.es/bibliotecas/MC/Rebeca

Recker, Astrid. From cultural heritage to digital knowledge: building infrastructures for a global knowledge society http://www.ariadne.ac.uk/issue59/ifla-3p-rpt/

Sede colaborativa del Consejo de Cooperación Bibliotecaria http://www.mcu.es/bibliotecas/MC/ConsejoCB/Presentacion.htm

Sistema de información sobre bibliotecas españolas http://www.mcu.es/bibliotecas/IN/estadisticas/index.html

María-Antonia Carrato-Mena, Subdirectora General de Coordinación Bibliotecaria, Ministerio de Cultura. Plaza del Rey, 1. 20004 Madrid. mariaantonia.carrato@mcu.es

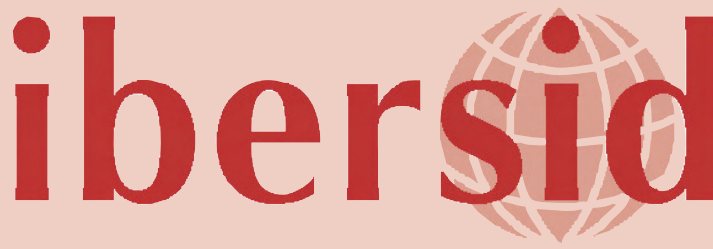

Zaragoza, 4-7 de octubre de 2010

\section{http://www.ibersid.org}

IBERSID es un foro internacional anual nacido en 1996 con el objetivo de promover la investigación entre académicos, investigadores y estudiantes de las Ciencias de la Documentación y de otras disciplinas relacionadas, interesados en identificar, analizar

y discutir de forma rigurosa, interdisciplinar, abierta y distendida los problemas profesionales y científicos.

La discusión se concentra en torno al concepto de sistemas de información y documentación como núcleo común alrededor del cual los distintos especialistas -archiveros, bibliógrafos, bibliotecarios, documentalistas, museólogos, gestores de la información y del conocimiento, 

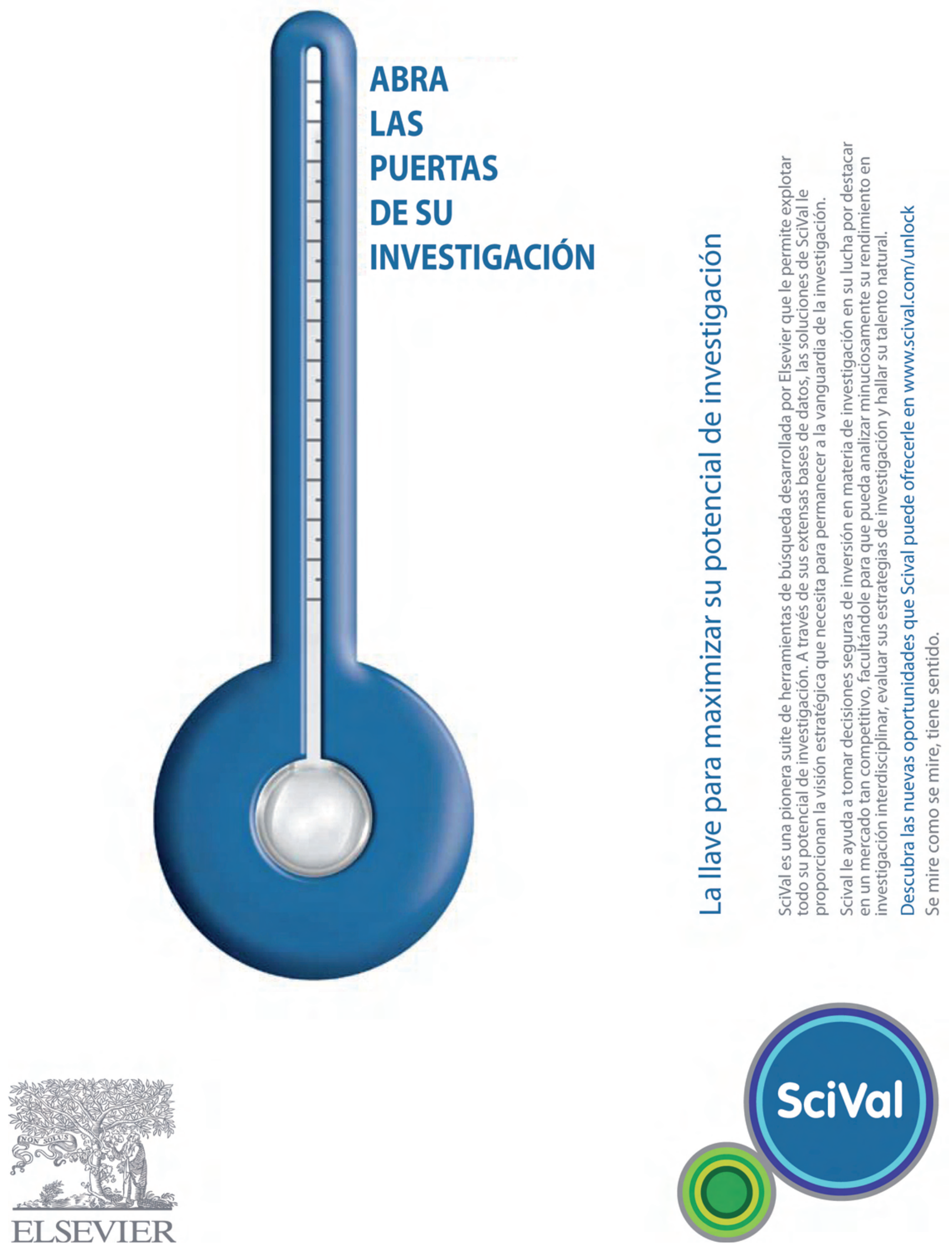

Scival 


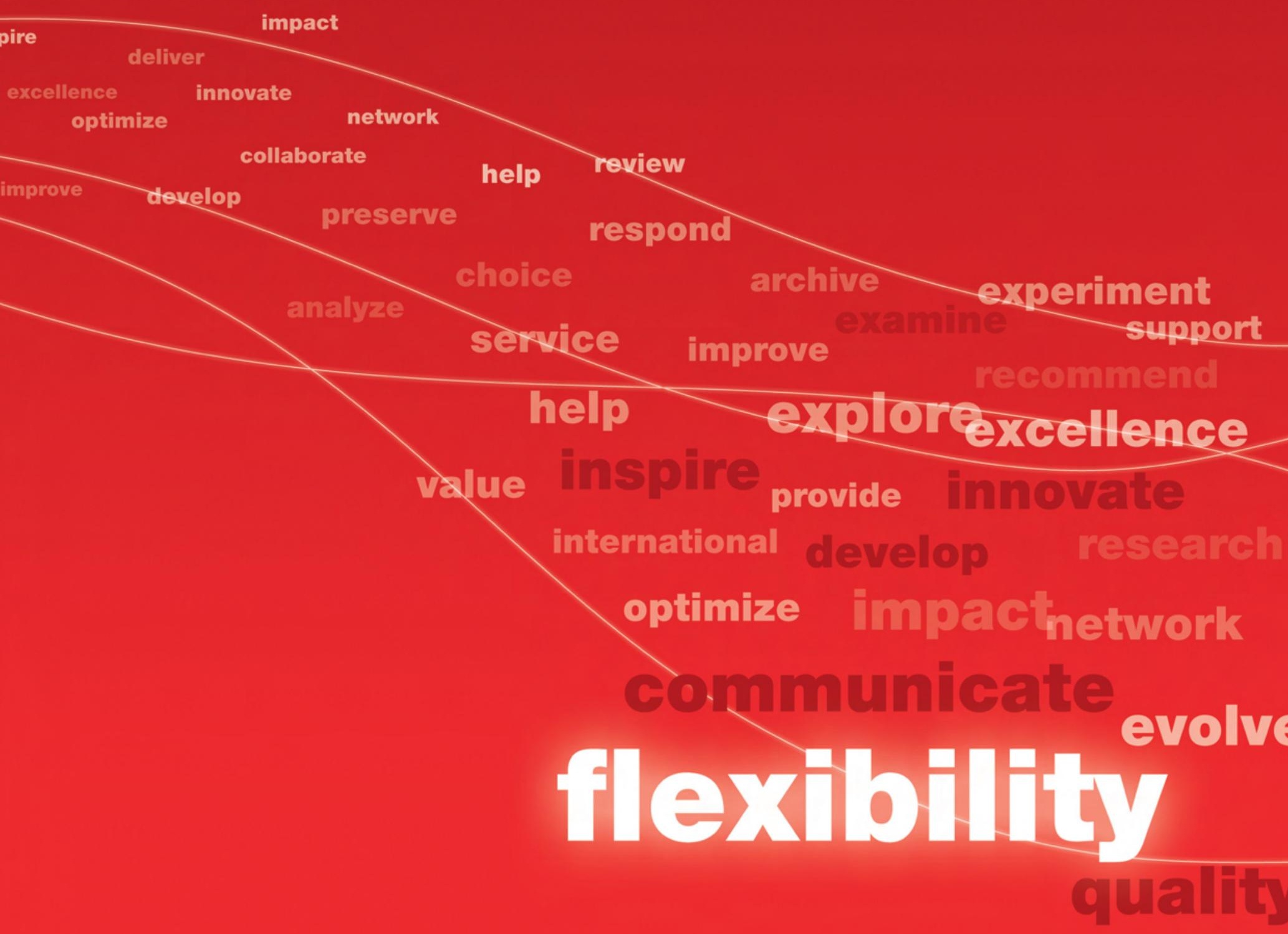

Licencia de acceso es el modelo flexible de NPG que le permite seleccionar las suscripciones más importantes para su institución. Elija entre publicaciones de alta calidad de Nature Publishing Group, incluyendo Scientific American y Palgrave Macmillan para crear una licencia que se ajuste a sus requerimientos y a las necesidades de sus usuarios. Total flexibilidad. Su elección.

Para mas informacion: www.nature.com/libraries/espanol 